\title{
Inspired Solutions that Harness the Power of the Cell
}

\begin{abstract}
We are global leaders in the design, manufacture, and application of gene editing and gene modulation, building cell models that harbour the genetics of human disease. These cells and the reagents used to build them - by us or our customers - are the basis of our broad range of products and services, providing researchers and drug developers the critical insights they need to drive the development and effective application of personalised medicines and cell and gene therapies.
\end{abstract}

\section{Introduction}

Drug development, from the earliest phases of research through to the clinic, has always been challenging. Under the standard approach, pharmaceuticals are developed on a 'one size fits all' basis, putting tens of thousands of potential drug compounds through a process that costs on average $\$ 2.6$ billion per approved drug, ${ }^{1}$ taking up to 15 years to complete.

Further, the traditional blockbuster model of drug development is no longer practical or sustainable, with the cost of R\&D in life science research falling to $3.2 \%$ among large cap pharma. ${ }^{2}$ As a result, life science researchers and drug developers are increasingly embracing a personalised medicine approach that uses genetic information to drive the development of genetically-targeted medicines.

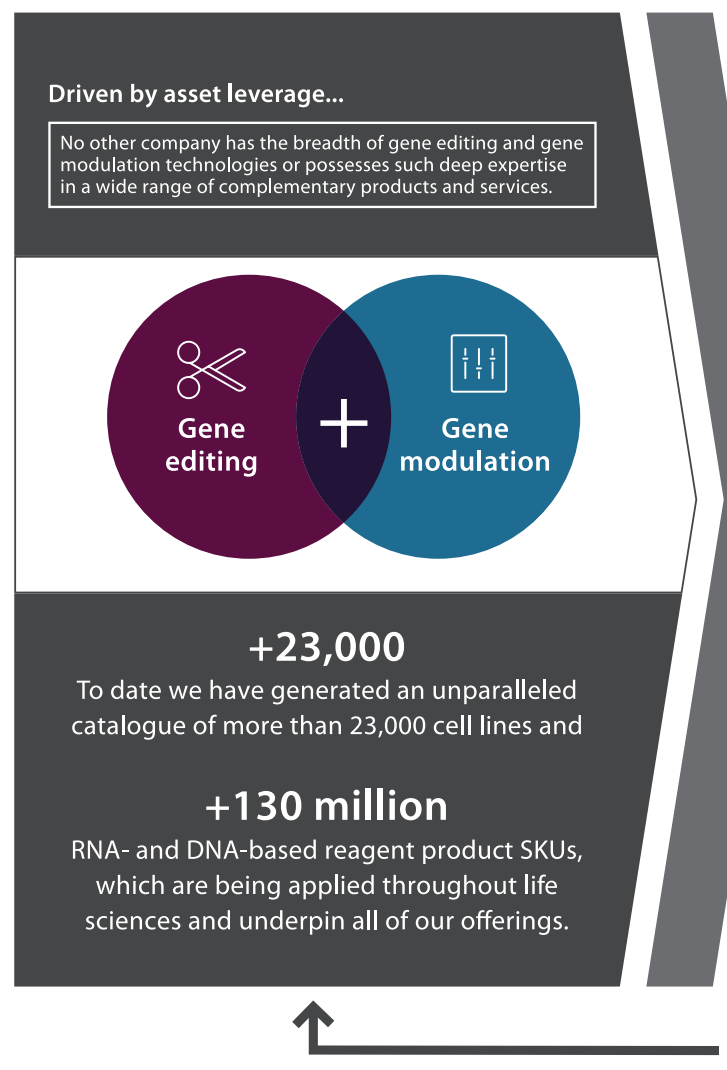

Fortunately, a wealth of genomic data is now emerging due to the low cost of sequencing. Whereas the first human genome cost just under $\$ 3$ billion to sequence, over the past decade the cost of human genome sequencing has rapidly fallen to just $\$ 1,000,{ }^{3}$ making it affordable and accessible for the broader research community. As a result, tens of thousands of human genomes are now sequenced every year and this information is transforming drug development programmes through the power of the cell.

\section{Horizon Provides 'Inspired Cell Solutions' - The Cell is at the Heart of All of Our Activities}

The cell lies at the heart of biology. Horizon's success is built upon our ability to modify the cell, helping
Custom model generation

Catalog cell and in vivo models

Reagent tools for customers to generate their own models
Disease models are applied in a range of highvalue contract research services.

Disease models are licensed for research use.

Cell lines are converted into additional products (e.g., reference standards). 
customers understand the foundations of cell biology, from basic research to exploring the key predictors and drivers of human disease, and translating that knowledge into valuable industrial applications through asset leverage.

We have a clear business focused on the cell, with commercial and research operations designed to ensure that everything we do is aligned around the effective deployment of the cell on behalf of our customers. We are meeting the needs of our customers today and anticipating their needs for tomorrow, in areas as diverse as personalised medicine, cell and gene therapy, and immuno-oncology.

Horizon has built a world-leading life sciences capability based on the delivery of essential products and services to four large customer groups with clear, unmet needs - academic research, drug development, biomanufacturing, and clinical diagnostics - which combined represent a $\$ 2.9$ billion market opportunity growing at approximately $20 \%$ per year.*

Horizon addresses these markets through a combination of research tools, cell lines, and derived products, and a range of services based on their application. Combined, our solutions were provided to more than 2,100 organisations around the world in 2017, which are deploying the power of the cell to improve the lives of patients.

\section{Horizon's Inspired Cell Solutions Support Customers Across the Entire Life Sciences Continuum}

\section{Cell Line Catalogue}

Horizon's Cell Line catalogue, at more than 23,000 cell line pairs, is the single largest bank of modified human cell lines available. These models help researchers understand how complex genetic diseases manifest themselves in patients and can reduce the cost of bringing to market new personalised therapies by streamlining many aspects of drug development including target identification, target validation, assay development, drug screening, lead optimisation and biomarker-driven clinical trial design. Our catalogue includes a wide range of genomic modifications such as point mutations, deletions, and insertions in an expanding range of cell lines that include common cancer lines, as well as our proprietary HAP1 haploid cell line.

\section{Research Reagents}

Horizon's research reagents product offering is new to the company, having been added to the product portfolio through the 2017 acquisition of Dharmacon. This catalogue of more than 130 million individual product variants enables researchers to do three things: to silence genes with the use of RNA interfer-

\footnotetext{
*Multiple sources, Horizon internal calculations.
}

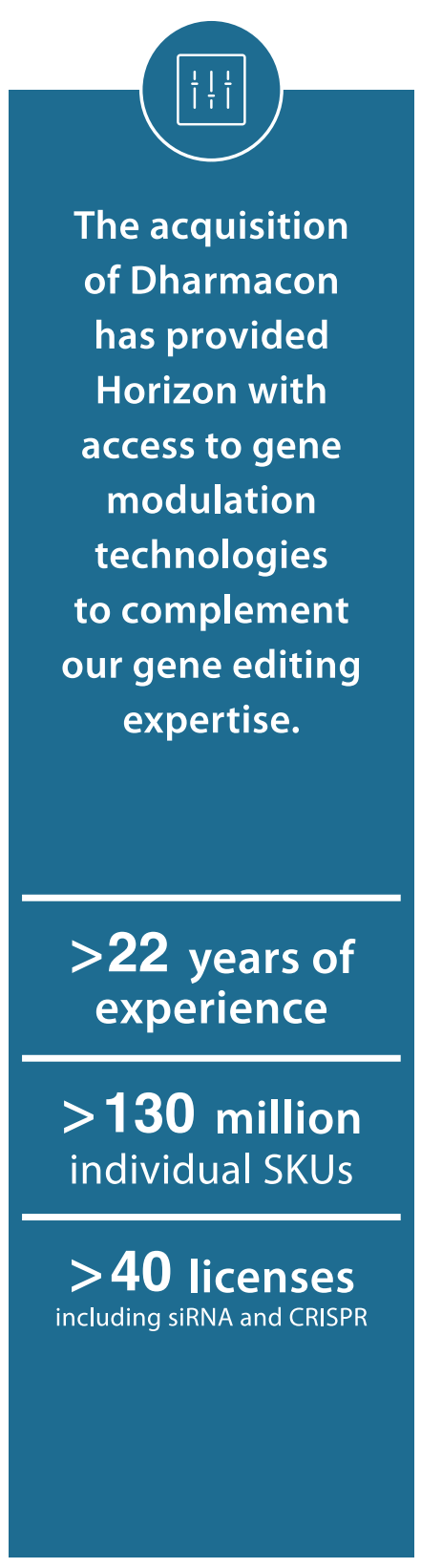

ence (RNAi); to express or overexpress genes through tools such as CDNA; and to permanently edit genes with CRISPR reagents.

These products are available to order individually, primarily through Dharmacon's eCommerce platform, or can be ordered in the form of libraries to support target identification and other forms of screening assays. This latter format has seen a particularly strong market response, with libraries placed at five of the top 20 pharmaceutical companies in 2017. Additionally, Dharmacon is renowned for its custom RNA synthesis capabilities, offers lentivirus manufacturing, and in 2017 launched the Edit-R CRISPRa reagent platform for genome-wide CRISPR activation. 


\section{In Vivo Models}

Horizon is a world-class provider of next-generation in vivo research models for specialised R\&D applications and to support preclinical drug development programmes. These models are available off the shelf, can be developed on demand to customers' specifications, or can be generated directly by our customers using reagents we provide. Our models are developed in partnership with the leading minds in ADMET, neuroscience, cardiovascular biology, immunology and oncology in order to ensure that they provide the most reliable and disease-relevant information possible.

\section{Bioproduction}

Biological therapeutics made up nine of the top ten best-selling drugs and represented an estimated $20 \%$ of the overall market in 2016 (up from $11 \%$ in 2002). ${ }^{4}$ The $\mathrm{CHO}$ cell line was first derived for bioproduction in 1956, however for decades efforts to improve its productivity was limited to what could be done with media and supplements. With the emergence of gene editing, however, fundamental improvements in the machinery of these drug factories could be made. Access to these improved bioproduction cell lines can be cost prohibitive, however, and their use is often restricted, stifling innovation. In 2014, Horizon started a 'CHO Revolution' with the goal of improving the efficiency and accessibility of biologic manufacturing for all participants in the industry, regardless of their size, by providing best in class cell lines available under flexible and affordable licensing terms.

\section{Molecular Diagnostics}

There are many potential sources of variability that can lead to molecular diagnostic tests providing incorrect or unreliable results, however as the leading innovator of cell line-derived Reference Standards, Horizon is changing this. Our Reference Standards offer a source of genetically defined, quantitative, sustainable and independent third-party reference material, critical to the validation and routine performance monitoring of assays and offering an unprecedented level of control. We provide our standards to customers through two models: by offering to vendors for the development and validation of their molecular assays with the goal of entering into OEM agreements for reliable highmargin revenue, and off-the-shelf through direct sales and our eCommerce platform.

\section{Cell and In Vivo Custom Model Generation}

With deep expertise in gene editing, and with access to multiple technologies in our gene editing platform, Horizon is able to make virtually any modification in a wide range of species and in almost any cell line including: our proprietary haploid human cells, a low-cost way for researchers to test their hypotheses; gene editing of iPSCs, an increasingly important tool to study disease biology; the modification of immune cells to investigate

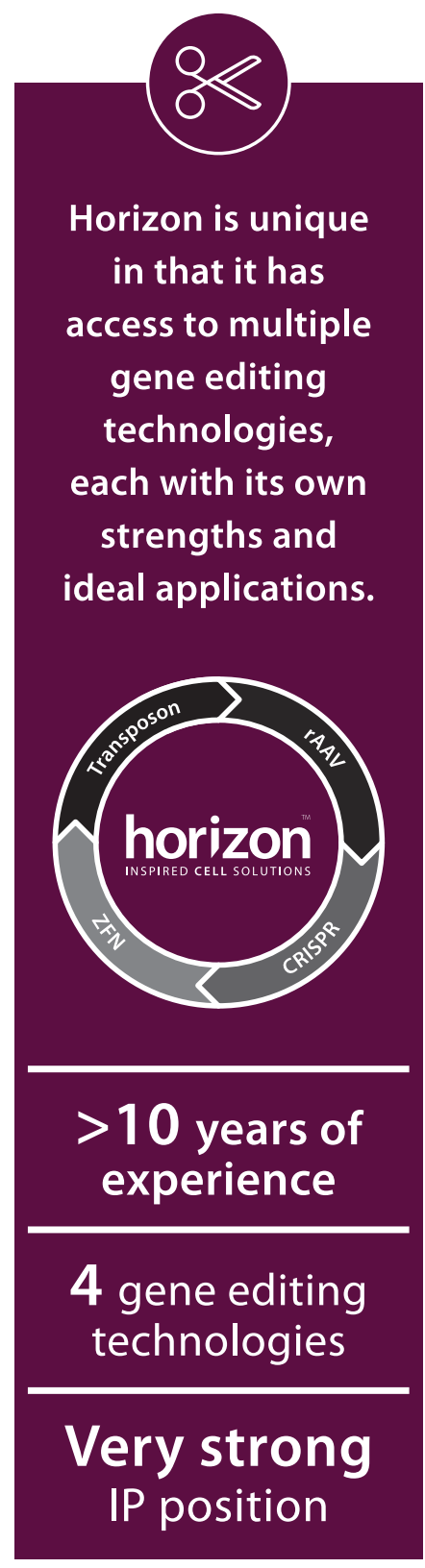

disease progression and to power immuno-oncology studies; and the custom modification of primary cells to support cell therapy programmes.

Over the more than ten years of our operation, Horizon has built a very strong reputation based not only on our success but also on the quality of our relationships. We partner with our customers, helping them to define the best model to drive their research, and do so with transparent pricing without the hidden costs that are common elsewhere in the industry.

\section{Assay Services}

Horizon's scientists are experts in the development and application of cell-based assays to support research 
programmes and drug discovery projects. We offer a range of specialised assays based on the use of our high value cell and in vivo disease models, which alongside our deep experience in the use of these models, provide high-value data to our customers. We work closely with customers under a partnership model so that the studies we perform address their key scientific questions, and we provide data interpretation where needed in order to maximise the impact the study has on the advancement of their programmes. Our Assay Services group is also responsible for the design and scale up of assays to high-throughput format.

\section{Genetic Screening}

Horizon has established a core expertise in Genetic Screening using gene trap, siRNA and CRISPR platforms. Using these techniques, we are able to explore the function of genes by interrupting their sequence or disrupting transcription by which they produce protein. In this way, these screens can be used to find and validate novel drug targets, to identify mechanisms of drug resistance or sensitivity, and to aid with patient stratification. Horizon performs these screens in both normal human cell lines and also in haploid cell lines. The Dharmacon acquisition has bolstered this already fast-growing part of the business by providing access in-house to their CRISPR and gene modulation libraries.

\section{Molecular Screening}

Combination therapies have become the standard of care in the treatment of many diseases, and so it is important to assess prospective new drugs to look for combinations that could result in increased efficacy, that could have a reduced effect, or that could be toxic when prescribed together. Horizon is a recognised leader in this area, with over a decade of experience analysing over 10 million unique drug combinations. We have also adapted this platform to build a groundbreaking platform for immuno-oncology drug discovery. Our collection of high throughput assays allows Horizon to rapidly identify single agents or combinations that effect $\mathrm{T}$-cell proliferation and polarisation, tumour cell lysis, and natural killer (NK) cell function on behalf of customers.

\section{References}

1. Mullard A. New drugs cost US $\$ 2.6$ billion to develop. Nature Rev. Drug Discovery 2014;13:877.

2. National Human Genome Research Institute. The Cost of Sequencing a Human Genome. Bethesda, MD: National Human Genome Research Institute, National Institutes of Health, 2016.

3. Terry C, Taylor K. A new future for R\&D? Measuring the return from pharmaceutical innovation 2017. London: Deloitte, 2018.

4. Lindsley CW. New 2016 Data and Statistics for Global Pharmaceutical Products and Projections through 2017. ACS Chem. Neurosci 2017;8:1635-1636. 